\title{
El portafolio como recurso didáctico en el proceso de aprendizaje del alumnado con trastorno por déficit de atención/hiperactividad
}

\section{Portfolios as a teaching resource in the learning process of students with attention-deficit/ hyperactivity disorder}

\author{
$M^{a}$ Teresa Martínez-Frutos ${ }^{1}$ \\ mariateresa.martinez12@um.es \\ Eva Herrera-Gutiérrez \\ evahg@um.es \\ Universidad de Murcia, España
}

\section{Resumen:}

En este trabajo realizamos una propuesta de portafolio como recurso educativo para atender algunas de las dificultades que presentan los niños con trastorno por déficit de atención/hiperactividad (TDAH) en la escuela. Partiendo de la conceptualización actual del trastorno y considerando las necesidades y problemas de estos alumnos en el ámbito académico, sugerimos un modelo de portafolio que facilite el trabajo coordinado familia-escuela, la organización de los contenidos de las materias escolares por parte del menor, su implicación y motivación por aprender, el desarrollo y expresión de la creatividad, así como el fomento y adquisición de hábitos de estudio. Aspectos todos ellos que favorecen el proceso de aprendizaje

\begin{abstract}
:
This article looks at portfolios as an educational resource to attend to some of the difficulties presented by school children with attention-deficit/hyperactivity disorder (ADHD). Based on the current conceptualization of this disorder and considering the needs and problems of these students within academic settings, we suggest a model of portfolio which facilitates coordinated work between families and schools, the organization of the contents of subjects by children themselves, their involvement and motivation in learning, the development and expression of creativity, as well as the generation and acquisition of study habits. All of these aspects will favor the learning process and will contribute to improving school perfor-
\end{abstract}

1 Dirección para correspondencia (correspondence address):

$M^{a}$ Teresa Martínez-Frutos. Universidad de Murcia. Departamento de Psicología Evolutiva y de la Educación. Facultad de Educación. Campus de Espinardo, s/n. 30100 Espinaro (España). 
El portafolio como recurso didáctico en el proceso de aprendizaje del alumnado con trastorno por déficit de atención/hiperactividad

Ma Teresa Martínez-Frutos y Eva Herrera-Gutiérrez

y contribuyen a la mejora del rendimiento escolar y la autoestima.

\section{Palabras clave:}

Trastorno por déficit de atención/hiperactividad (TDAH); conceptualización; necesidades especiales; portafolio; proceso de aprendizaje. mance and self-esteem.

\section{Keywords:}

Attention-deficit/hyperactivity disorder (ADHD); conceptualization; special needs; portfolio; learning process.

\section{Résumé:}

Dans cet article, nous faisons une proposition de portefeuille comme une ressource éducative pour répondre à certaines des difficultés présentées par les enfants atteints d'un trouble déficit de l'attention/hyperactivité (TDAH) à l'école. Basé sur la conceptualisation actuelle de la maladie et compte tenu des besoins et des problèmes de ces élèves dans le domaine académique, nous vous proposons un modèle de portefeuille pour faciliter le travail coordonné famille-école, l'organisation du contenu des matières scolaires par l'enfant, son implication et la motivation pour l'apprentissage, le développement et l'expression de la créativité, ainsi que la promotion et l'acquisition d'habitudes d'étude. Tous les aspects favorisent le processus d'apprentissage et contribuent à l'amélioration du rendement scolaire et l'estime de soi.

\section{Mots clés:}

Trouble déficit de l'attention/hyperactivité (TDAH); conceptualisation; besoins spéciaux; portefeuille; processus d'apprentissage.

Fecha de recepción: 9-1-2016

Fecha de aceptación: 28-5-2016

\section{Introducción}

El trastorno por déficit de atención/hiperactividad (TDAH) es un síndrome neurobiológico de etiología diversa. Está relacionado con una alteración en la producción cerebral de dos neurotransmisores, dopamina y noradrenalina, que genera problemas en los circuitos reguladores de varias zonas del cerebro (Barkley, 2010).

Este trastorno se caracteriza por los síntomas clave de desatención, hiperactividad e impulsividad. Se inicia en la infancia con un patrón de comportamiento característico, destacando la dificultad para prestar atención a los detalles, las tareas y actividades, la impulsividad, los problemas de organización y la incapacidad para permanecer sentado y en silencio en situaciones que lo requieren, entre otras dificultades. Estos patrones de conducta se manifiestan principalmente en la escuela y en el hogar y pueden dar lugar a otros problemas asociados si no son tratados adecuadamente.

El tratamiento considerado más apropiado y efectivo para los niños 
con trastorno hiperactivo es el multicomponente o multidisciplinar (Herrera-Gutiérrez, Bermejo, Fernández, \& Soto, 2011; Herrera-Gutiérrez, Calvo, \& Peyres, 2003; Lasa, 2007; Montoya \& Herrera-Gutiérrez, 2014). Desde este enfoque de abordaje integral, que defiende las terapias combinadas, se debe intervenir y atender los aspectos cognitivos, conductuales, educativos, afectivos, familiares y sociales. Es por ello que se requiere una intervención en la que estén implicados varios profesionales (médico, psicólogo, profesor, etc.), los padres y demás personas del entorno próximo al sujeto con este diagnóstico.

El TDAH es el trastorno del comportamiento más diagnosticado en la infancia. Se estima que afecta entre un $5 \%$ y un $7 \%$ de los niños en edad escolar, dándose más en varones (APA, 2000, 2013). Dada la frecuencia con la que se presenta y las dificultades que ocasiona a los menores con este diagnóstico, resulta fundamental que los educadores estén familiarizados con los síntomas y los problemas que muestran estos alumnos en el aula, para saber identificarlos a tiempo y poder poner en marcha las pautas y actuaciones necesarias para ayudar a compensar sus invisibles dificultades.

De este modo, el profesor deberá conocer y emplear todas aquellas técnicas y estrategias metodológicas que ayuden a la mejora del rendimiento académico y funcionamiento escolar, emocional y social de este tipo de estudiantes. La finalidad de este trabajo es mostrar los beneficios que puede aportar el portafolio como instrumento educativo capaz de favorecer el proceso de aprendizaje y la coordinación entre el profesorado y las familias de los niños con trastorno de hiperactividad.

\section{Conceptualización actual y respuesta educativa a los problemas de déficit de atención e hiperactividad}

\section{Trastorno por déficit de atención/hiperactividad}

La definición de la alteración que hoy conocemos como TDAH ha ido evolucionando según el síntoma al que se le ha dado mayor relevancia en los estudios realizados. Surge un modelo médico inicial que hace hincapié en las causas genéticas, biológicas y neurológicas del trastorno (Kahn \& Cohen, 1934; Strauss \& Lehtinen, 1947; Wender, 1971).

Posteriormente, otros autores (Ross \& Ross, 1982; Rutter, 1989) aban- 
donan la posición centrada en el déficit neurológico, más o menos grave, para insistir en otras causas de índole más psicológica y comportamental. Sus investigaciones proponen una línea de intervención fundamentada en la aplicación de técnicas conductistas cuyo objetivo será disminuir el nivel de actividad motora e impulsividad y mejorar el nivel de atención. Además, se crean programas basados en un entrenamiento cognitivocomportamental.

En la actualidad, estamos asistiendo al resurgir del modelo médicoorganicista del TDAH (Herrera-Gutiérrez, 2015). Los estudios ponen el énfasis en el origen neurobiológico de este trastorno y en la dificultad, biológicamente mediada, de las personas que lo presentan para activar y sostener aquellas funciones responsables de la autorregulación de la conducta (Aguilar-Domingo, Herrera-Gutiérrez, \& Gómez-Amor, 2013; Barkley, 2005; Mulas, Mattos, de la Osa-Langreo, \& Gandía-Benetó, 2007; Sharma \& Couture, 2014).

Para su diagnóstico, entre otros elementos y pruebas que lo facilitan, se deben contemplar los criterios diagnósticos especificados en el DSM-5 (APA, 2013), teniendo en cuenta que existen tres presentaciones clínicas distintas del trastorno, caracterizadas por los síntomas (criterio A) que seguidamente detallamos.

- Presentación predominante con falta de atención, consistente en la manifestación de 6 o más síntomas de inatención (sólo 5 a partir de los 17 años de edad) durante al menos 6 meses, en un grado que no concuerda con el nivel de desarrollo y que afecta directamente a las actividades sociales y académicas/laborales de la persona:

- No presta atención a los detalles o comete errores por descuido.

- Tiene dificultad para mantener la atención en actividades recreativas.

- Parece no escuchar cuando se le habla directamente.

- Tiene dificultad para seguir las instrucciones hasta el final y acabar los quehaceres.

- Muestra dificultad con la organización de tareas y actividades.

- Evita o le disgustan las tareas que requieren un esfuerzo mental sostenido.

- Pierde cosas necesarias para realizar las actividades.

- Se distrae con facilidad.

- Se olvida y descuida las tareas diarias. 
- Presentación predominante hiperactiva/impulsiva, donde el individuo muestra 6 o más síntomas de hiperactividad e impulsividad (es suficiente 5 desde los 17 años) durante al menos 6 meses, en un grado que no corresponde al nivel de desarrollo y que repercute manifiestamente en las actividades sociales y académicas/laborales:

- Mueve nerviosamente o juega con las manos o los pies o se retuerce en el asiento.

- Se levanta en situaciones en que se espera que permanezca sentado.

- Corre o trepa en escenarios o momentos en los que resulta inapropiado (en adolescentes o adultos puede limitarse a estar inquieto).

- Tiene dificultad para jugar o realizar actividades recreativas tranquilamente.

- Se mantiene ocupado y actúa como si estuviera impulsado por un motor.

- Habla en exceso.

- Responde antes de que se haya terminado de formular las preguntas.

- Muestra dificultad para esperar su turno.

- Interrumpe o se inmiscuye en conversaciones o en actividades de los demás.

- Presentación combinada, basada en la presencia de los síntomas de inatención y de hiperactividad-impulsividad propios de las dos presentaciones anteriores, al menos durante los últimos 6 meses.

Además, para realizar el diagnóstico, debe cumplirse que: 1) algunos síntomas de inatención o hiperactivo-impulsivos estén presentes antes de los 12 años (criterio B); 2) varios se den en dos o más contextos de la vida cotidiana, por ejemplo en el hogar y en la escuela o trabajo (criterio C); 3) Ileguen a obstaculizar el funcionamiento social, académico o laboral o reduzcan su calidad (criterio D); y 4) no se produzcan exclusivamente durante el curso de otros trastornos o problemas mentales que puedan estar justificando la sintomatología observada (criterio E). 
El portafolio como recurso didáctico en el proceso de aprendizaje del alumnado con trastorno por déficit de atención/hiperactividad

Ma Teresa Martínez-Frutos y Eva Herrera-Gutiérrez

\section{Necesidades especiales}

El TDAH comporta una deficiencia significativa para mantener la atención, controlar los impulsos y demostrar una conducta ajustada a las reglas o normas establecidas (obediencia, autocontrol y resolución de problemas).

Según Barkley (2010), las principales dificultades y necesidades que presentan los alumnos con este trastorno en la escuela no surgen de una falta de habilidad, sino de un déficit de autorregulación. Afirma que los sistemas cerebrales responsables del autocontrol y la voluntad no funcionan o lo hacen de manera inadecuada, por lo que mantener unos niveles normales en estas dos habilidades básicas les resulta muy difícil.

A este respecto, Tirapu, Muñoz, Pelegrín y Albéniz (2005) muestran cómo las funciones ejecutivas (responsables del control y la inhibición del pensamiento y la acción), entendidas como solución de problemas, requieren procesos de selección de objetivos, planificación y monitorización. Las funciones ejecutivas son las que permiten focalizar la atención y rechazar estímulos irrelevantes, sosteniendo la actividad cognitiva sobre una determinada tarea o estímulo durante un período de tiempo.

Sin embargo, el déficit en el autocontrol que presentan los estudiantes con TDAH genera una serie de dificultades y disfunciones en este ámbito que un profesional de la enseñanza debe conocer antes de enfrentarse a la labor educativa. Así, el docente debe saber que en estos niños el inadecuado funcionamiento de las funciones ejecutivas se relaciona con innumerables dificultades: inapropiada respuesta inhibitoria; problemas para controlar los impulsos y demorar recompensas; exceso de actividad en tareas irrelevantes o escasa regulación de la actividad frente a las exigencias de una determinada situación; dificultades en el control de las emociones, la motivación y la alerta; alta variabilidad en el rendimiento del trabajo; elevada distraibilidad y problemas para mantener la atención de forma sostenida; además de un rendimiento académico deficitario (por debajo de su capacidad).

La investigación realizada (Arcia, Frank, Sánchez-LaCay, \& Fernández, 2000; Barkley, 2008; Hidalgo \& Soutullo, 2006; Miranda, Presentación, Colomer, \& Roselló, 2011; Orjales, 2007; Scandar, 2000) ha señalado varios problemas en el proceso de aprendizaje de estos alumnos, tales como la dificultad para controlar los procesos de atención, las limitaciones para seguir detenidamente las instrucciones ante una 
tarea y las dificultades en el manejo de la memoria de trabajo verbal y no verbal.

Asimismo, en numerosas ocasiones se ha puesto de manifiesto la asociación del comportamiento hiperactivo con resultados académicos bajos y una mayor inadaptación escolar y social (Eisenberg \& Schneider, 2007; Loe \& Fieldman, 2007; Sánchez-Mármol \& Herrera-Gutiérrez, 2010).

Por su parte, Barkley $(2002,2008)$ indica que los problemas de comportamiento llegan a provocar hasta un $15 \%$ de las expulsiones de los niños con TDAH del colegio, observándose una elevada tasa de fracaso escolar entre la población así diagnosticada.

Si bien en ocasiones se olvida y, por ello, las necesidades de estos alumnos no son satisfechas por el sistema educativo, en el contexto escolar es preciso tener presente que los síntomas principales del trastorno hiperactivo dificultan el desempeño académico y el comportamiento de los menores con esta alteración.

La adecuada detección e intervención desde un enfoque multidisciplinar (educativo, psicológico, social, médico) puede reducir y prevenir las consecuencias negativas de este trastorno en el individuo y en su entorno, así como el desarrollo de otros desórdenes secundarios, como el trastorno negativista desafiante, el trastorno disocial, los problemas emocionales, las adicciones y los trastornos del aprendizaje, entre otros.

Además, es importante advertir que, junto a las necesidades especiales, en la persona con TDAH son muchos los aspectos positivos de su personalidad, tales como su curiosidad, creatividad y espontaneidad (Barkley, 2006), en los que podemos apoyarnos para avanzar en la aventura de aprender.

\section{Respuesta educativa}

Dadas las características de este trastorno, en el que hemos visto que destacan la impulsividad, los problemas de atención y las alteraciones comportamentales, el profesor deberá conocer y emplear todas aquellas estrategias y técnicas que ayuden a la mejora de estas manifestaciones y que puedan ser aplicables en el ámbito educativo.

El docente se convierte en una pieza fundamental a la hora de enseñar al niño a organizarse en el entorno escolar. Su función es muy importante para apoyar el proceso de aprendizaje del alumno con tras- 
El portafolio como recurso didáctico en el proceso de aprendizaje del alumnado con trastorno por déficit de atención/hiperactividad

Ma Teresa Martínez-Frutos y Eva Herrera-Gutiérrez

torno de hiperactividad y enseñarle estrategias que le permitan organizar los contenidos curriculares, las actividades y los materiales y, de esta forma, ayudarle a superar los objetivos de las diversas materias. Aunque al principio suponga un esfuerzo considerable y una cantidad de tiempo adicional, a largo plazo los beneficios serán considerables tanto para el profesor como para el alumno. Una vez que el niño asimile las estrategias enseñadas, como una rutina más de su vida escolar, le resultará más fácil cumplir con sus obligaciones.

Igualmente, los profesores han de utilizar sistemas que no penalicen, y por tanto no paralicen, y que sean atractivos, innovadores y activos (por ejemplo, las tecnologías de la información y la comunicación). Como señala Gamo (2012), un buen docente tiene que hacer el traje educativo a la medida del niño utilizando para ello metodologías compensatorias e innovadoras.

A este propósito, conviene recordar la obligación del sistema educativo de velar por la calidad y la equidad de la educación. La Ley Orgánica 2/2006, de 3 de mayo, de Educación (LOE) y la Ley Orgánica 8/2013, de 9 de diciembre, para la Mejora de la Calidad Educativa (LOMCE) establecen que los centros educativos deben articular los procedimientos y recursos necesarios para identificar y atender tempranamente las necesidades educativas de cualquier índole que presente un alumno.

Así, se busca garantizar que todos los menores alcancen el máximo desarrollo personal, intelectual, social y emocional, junto con los objetivos académicos. Sin olvidar la importancia y necesidad de regular la participación de los padres o tutores en las decisiones que se tomen, así como de asesorarles adecuadamente en todo lo que afecte a la educación de sus hijos.

A nuestro juicio, se trata de que la escuela proponga objetivos, metodologías, estrategias y recursos de atención integral (Herrera-Gutiérrez, 2015), que promuevan el desarrollo del niño con TDAH en su totalidad y, por tanto, además de alcanzar los objetivos meramente académicos, permitan también que adquiera las habilidades de manejo emocional y el despliegue de su resiliencia. Todo ello desde el convencimiento de que la adopción de este enfoque favorecerá no sólo el aprendizaje y desarrollo del menor con trastorno hiperactivo sino del conjunto del alumnado. 


\section{Protocolos y procedimientos de atención integral del TDAH}

En España algunas Comunidades Autónomas, como Canarias, Murcia, y Castilla y León (Artiles \& Jiménez, 2006; Consejería de Sanidad y Consumo - Consejería de Educación, Formación y Empleo, Región de Murcia, 2012; Láez, Requejo, Silvano, \& Velasco, 2011), han elaborado protocolos que recogen las actuaciones educativas y sanitarias para la detección, diagnóstico e intervención en el TDAH.

Además de facilitar el trabajo conjunto y la coordinación de los distintos profesionales implicados, estos protocolos son guías de atención educativa que facilitan que el profesorado pueda conocer con más exactitud qué es el TDAH, qué procedimientos y estrategias metodológicas pueden emplearse en el aula y algunas medidas a tener en cuenta a la hora de llevar a cabo la evaluación de estos niños.

Asimismo, disponemos de una gran variedad de estrategias y técnicas para abordar las dificultades que presenta el alumnado con TDAH en el contexto escolar, como las que seguidamente indicamos, que han sido señaladas por diversos autores (Barkley, 2008; Martínez-Frutos, HerreraGutiérrez, \& López-Ortuño, 2014; Miranda, 2011; Orjales, 2002; Scandar, 2000; Soutullo, 2004).

a) Procedimientos para mejorar los síntomas de déficit de atención:

- Sentar al alumno próximo al docente y avisarle de su distracción cuando sea necesario.

- Disminuir posibles estímulos distractores (ruidos, luces...) y evitar sobreestimular el aula y las actividades.

- Enseñarle estrategias para planificar y gestionar mejor su tiempo y actividades (por ejemplo, la preparación y realización de exámenes).

- Darle instrucciones breves y sencillas (evitando información irrelevante) y pedirle tareas concretas, claras y razonables.

- Establecer hábitos y rutinas diarias (anotar tareas en la agenda, relajación en horas concretas, etc.).

- Fraccionar las actividades en pequeñas partes o etapas.

- Proponer tareas atractivas que incrementen la motivación por aprender.

- Emplear recompensas inmediatas que promuevan la automotivación.

- Desarrollar hábitos favorecedores del aprendizaje y técnicas de 
estudio que le ayuden a comprender qué tiene que hacer y cómo tiene que hacerlo.

- Transmitir retroalimentación positiva, destacar habilidades positivas, reforzar el afán de superación, la motivación por el logro y la mejora continua; estrategias todas ellas que favorecen la autoestima.

\section{b) Técnicas para reducir las manifestaciones de hiperactividad:}

- Procurar que el alumno se siente y trabaje junto a compañeros tranquilos que pueden ayudarle a supervisar las tareas y actuar como modelos de conducta.

- Al comenzar a trabajar guiarle a que se marque un objetivo posible de alcanzar, facilitando así su motivación y concentración.

- Secuenciar las actividades de manera que se alternen los periodos que exijan mantener la atención y otros que permitan un poco de movimiento y distensión, contribuyendo a la asimilación progresiva de la información.

- Emplear métodos de enseñanza activos y participativos (aprendizaje cooperativo, tutoría entre iguales, enseñanza recíproca, etc.).

- Dar contingencias adecuadas a sus conductas, reforzando las adecuadas (trabajo silencioso) e ignorando las inadecuadas (exceso de actividad).

- Favorecer la participación y estimular la responsabilidad del alumno en su proceso de aprendizaje.

c) Estrategias para mejorar los síntomas de impulsividad:

- Especificar con claridad y recordar las normas.

- Empatizar con el alumno y mostrar confianza y comprensión ante sus necesidades.

- Enseñarle técnicas de autocontrol y relajación.

- Analizar y describir las conductas que hay que mejorar y tratar con técnicas de modificación de conducta (contrato de contingencias, moldeado, economía de fichas, etc.).

- Utilizar el entrenamiento en autoinstrucciones que favorece la verbalización, reflexión y evaluación de su desempeño en las actividades.

- Emplear técnicas de resolución de conflictos que faciliten la reflexión y comprensión del comportamiento propio y ajeno. 


\section{d) Recomendaciones para evaluar el rendimiento:}

- Sobre todo en las primeras etapas educativas, realizar una evaluación global, continua y formativa, donde la observación directa y sistemática y el análisis de las producciones diarias del alumno constituyan las técnicas principales del proceso de evaluación.

- Procurar evaluar criterialmente (en términos de logro) y evitar poner una calificación numérica a todos los trabajos y producciones.

- Entender la evaluación como un espacio de aprendizaje, ofreciendo un feedback de calidad y atendiendo más al proceso que al resultado.

- Efectuar una evaluación personalizada, que tenga en cuenta los conocimientos iniciales y las posibilidades del alumno.

- Evaluar no solo los progresos y objetivos logrados, sino también los recursos utilizados, los espacios, la metodología y las actividades propuestas para el aprendizaje.

- Evaluar únicamente con exámenes tradicionales al alumnado con TDAH puede resultar poco inclusivo y dudosamente objetivo. En todo caso, al realizar este tipo de pruebas se deberían tener en cuenta las siguientes indicaciones:

1. Informar con toda la antelación posible de las fechas de exámenes y demás trabajos objeto de evaluación.

2. No fijar más de un examen por día, ni concentrar muchos en una misma semana. Tampoco incluir parciales aprobados en un nuevo examen.

3. Evitar pruebas largas y extensas. Reducir el número de preguntas y hacerlas más cortas. Es preferible realizar exámenes de poco contenido y con más frecuencia (por ejemplo, un sólo tema en primaria y primeros cursos de secundaria).

4. En los exámenes y demás pruebas académicas conceder más tiempo al alumno e ir avisándole del que le va quedando.

5. Leer en voz alta los enunciados de las preguntas y comprobar que los comprende.

6. Si no logra centrarse en lo que se le está preguntando, ayudarle a reconducir su atención.

7. Antes de entregar el examen o prueba escrita recordarle que lo revise y supervisar que ha contestado a todas las preguntas 
El portafolio como recurso didáctico en el proceso de aprendizaje del alumnado con trastorno por déficit de atención/hiperactividad

Ma Teresa Martínez-Frutos y Eva Herrera-Gutiérrez

(son alumnos que suelen olvidar apartados aunque sepan la respuesta).

8. Dar la posibilidad de terminar o completar exámenes inconclusos mediante exposiciones orales.

\section{El portafolio como recurso para el aprendizaje del alumnado con dificultades de atención e hiperactividad}

García (2000) describe el portafolio como una colección de trabajos, realizados por el alumno o el profesor, que se relacionan directa o indirectamente con actividades vinculadas a contenidos curriculares. Así, podríamos considerar el portafolio educativo como una carpeta donde se van recogiendo las notas, resúmenes, actividades y reflexiones de todo el proceso educativo, referido a una asignatura y habitualmente con un orden cronológico.

El portafolio, a modo de dossier que contiene los trabajos más representativos del alumno en un área determinada, se emplea en EEUU como alternativa o como complemento a los exámenes tradicionales. Uno de los más conocidos es el Portfolio Europeo de las Lenguas (PEL), propuesto por el Consejo de Europa a los distintos estados para la aplicación del Marco Común Europeo de Referencia para las Lenguas (MCERL). El PEL se utiliza como un cuaderno donde el estudiante realiza anotaciones y reflexiones de las lenguas que usa o aprende. Está destinado a todos los europeos mayores de 3 años, presentándose con formato diferente según la edad y el nivel educativo (Cassany, 2002, 2006).

En el campo de la educación, el portafolio se ha convertido en un recurso de provecho para la enseñanza y la evaluación. Se ha destacado su utilidad como procedimiento de evaluación de la trayectoria del aprendizaje (Klenowski, 2005) y su contribución al desarrollo de las competencias establecidas en el Espacio Europeo de Educación Superior (Colas, 2005).

Precisamente en esta dirección, González (2015) señala que hay que fomentar el uso del portafolio en los centros escolares para propiciar el aprendizaje autónomo del estudiante. Además, este recurso educativo sirve al docente como herramienta de evaluación individual de las competencias del alumnado. Asimismo, su práctica permite que tanto profesores como alumnos puedan reflexionar y analizar los contenidos y los objetivos alcanzados. 
Cuando el portafolio es digital la recopilación de trabajos del estudiante se realiza utilizando las Tecnologías de la Información y la Comunicación (TIC), lo que posibilita la inclusión de imágenes, vídeo y sonido, además del formato escrito (González, 2014). Sin duda, la incorporación de las TIC aporta numerosas posibilidades a los portafolios. En primer lugar, su versatilidad, flexibilidad e interactividad permiten al estudiante aprender a planificarse y autogestionarse mejor y, en segundo lugar, que se convierta en productor de información y que la comparta con el resto del mundo de forma inmediata.

Si nos centramos en el alumnado con necesidades especiales el interés del portafolio puede llegar a ser incluso mayor. La atención educativa a los estudiantes con TDAH exige del profesorado un planteamiento diferenciado en la utilización de metodologías y en la organización del aula. El centro debe ser consciente de que los alumnos con esta alteración aprenden de un modo diferente y por tanto es necesario introducir cambios. En este sentido, el portafolio puede servir para alcanzar múltiples objetivos de aprendizaje, además de posibilitar la inclusión de diferentes tipos de actividades, facilitar la adquisición de hábitos de estudio, favorecer la comunicación y el trabajo coordinado familia-escuela y fomentar la autogestión.

Igualmente, el portafolio puede favorecer la comunicación en las familias, entre padres e hijos, en lo referente al ámbito escolar. Así concebido, es un instrumento que posibilita a ambos tener acceso con suficiente antelación a las fechas previstas para la entrega de trabajos y demás actividades académicas, tal y como muestra nuestra propuesta de portafolio (véase el apartado 7, calendario y planificación de tareas), lo que ayudará a planificar el tiempo de estudio y realizar la organización y supervisión necesarias.

La Orden ECD/65/2015, de 21 de enero, por la que se describen las relaciones entre las competencias, los contenidos y los criterios de evaluación de la educación primaria, la educación secundaria obligatoria y el bachillerato, reitera la necesidad de que el profesorado se implique en la producción y diseño de diferentes tipos de materiales, adaptados a los variados niveles, estilos y ritmos de aprendizaje de los estudiantes, con la finalidad de dar respuesta a la diversidad en el aula e individualizar los procesos de construcción de los aprendizajes. También señala que se debe promover la utilización de una variedad de materiales y recursos didácticos, destacándose el uso del portafolio. Este último se considera 
El portafolio como recurso didáctico en el proceso de aprendizaje del alumnado con trastorno por déficit de atención/hiperactividad

Ma Teresa Martínez-Frutos y Eva Herrera-Gutiérrez

que proporciona información extensa sobre el proceso de aprendizaje de los estudiantes, facilita y refuerza la evaluación continua y posibilita compartir resultados de aprendizaje. Igualmente, propone este recurso como herramienta motivadora para el alumnado, que fomenta su autonomía y estimula su capacidad de reflexión y crítica.

Así pues, considerando tanto las posibilidades como las dificultades que presentan los menores con TDAH, como recurso educativo, el portafolio reúne numerosas ventajas, contribuyendo a cubrir diversas funciones (véase la Figura 1). Además de servir para la detección y evaluación de las potencialidades y necesidades, permite que los niños aprendan de manera más consciente y se impliquen activamente en el aprendizaje. También ayuda a que tengan más claros los objetivos a alcanzar, posibilita que identifiquen y organicen de manera más efectiva los contenidos y actividades de las materias a las que se destine, fomenta la reflexión, incrementa la responsabilidad y favorece el desarrollo de la creatividad y la mejora del rendimiento académico y de la autoestima.

La creatividad, entendida como un reto para la educación del siglo XXI (Klimenko, 2008), conlleva potenciar en el aula la curiosidad, la imaginación, la capacidad de observación, de asumir riegos y de dar respuestas novedosas a los problemas que se plantean. No tener en cuenta las peculiaridades del alumnado y no fomentar la creatividad y el estilo personal puede ser perjudicial para el aprendizaje, pues trae como consecuencia la estandarización del rendimiento (Klenowski, 2005). Se precisan, en consecuencia, procedimientos de evaluación alternativos, que enfaticen más las fortalezas que las debilidades y más enfocados a documentar el crecimiento del individuo en un determinado periodo de tiempo, en lugar de comparar entre sí a los estudiantes (López \& Hinojósa, 2000).

En este escenario, el portafolio nos brinda la oportunidad de alejarnos de una enseñanza tradicional y uniformadora, respetando la individualidad y la riqueza de las diferencias personales en el proceso de enseñanza-aprendizaje. De acuerdo con esto, el modelo de portafolio que aquí planteamos, en sus diferentes apartados, persigue contribuir a desarrollar la creatividad del alumno y su capacidad de motivación y autorregulación y, por tanto, a mejorar su rendimiento académico. 


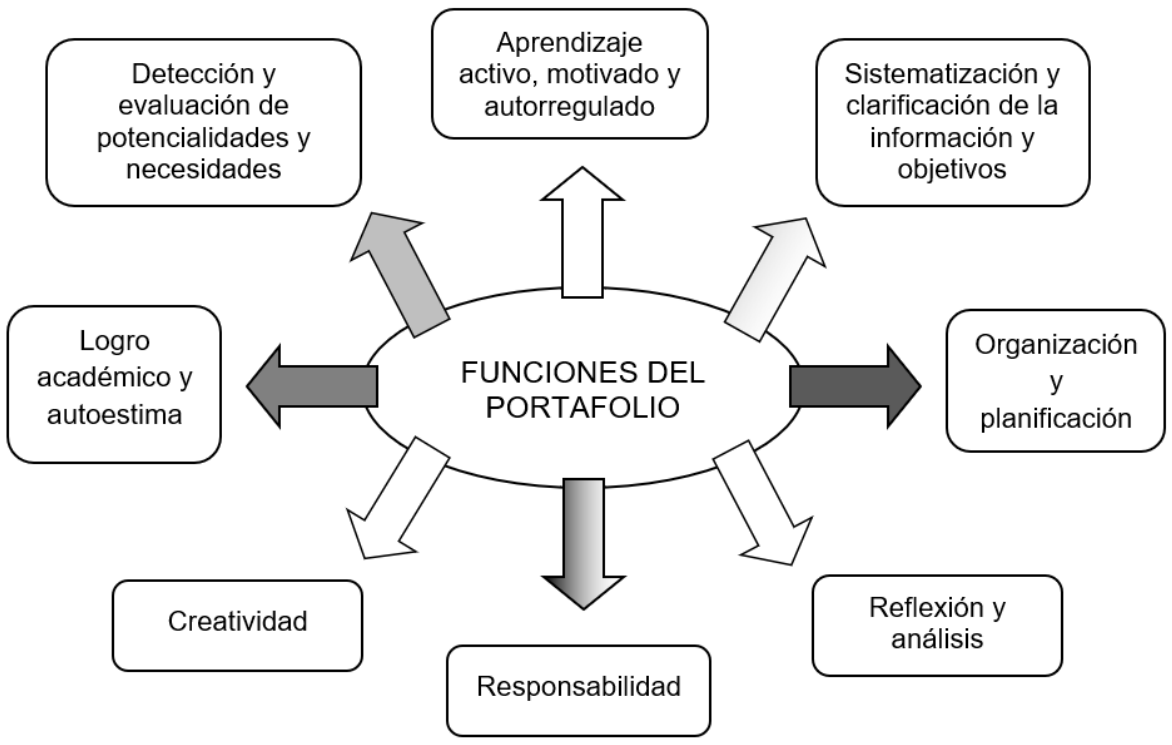

Figura 1. Funciones del portafolio de especial interés en alumnos con trastorno hiperactivo.

Teniendo en cuenta la gran flexibilidad que caracteriza a este instrumento, en referencia a los apartados generales o secciones que se podrían incluir en el portafolio, destacamos las que, por sus implicaciones didácticas y psicoeducativas, consideramos imprescindibles:

1) Presentación e índice. Es un apartado introductorio, a modo de presentación, con los datos de identificación y demás información específica (intereses y otras peculiaridades) del estudiante y los elementos principales incluidos en el portafolio (índice de contenidos).

2) Diario académico. Es el sitio donde cada día se anota la fecha y los enunciados de los contenidos trabajados en el aula (explicaciones del profesor, tareas realizadas, etc.). Sirve para que el alumno recuerde qué ha aprendido y cuándo ha sucedido.

3) Esquemas y resúmenes. En esta sección se incluyen breves anotaciones y esquemas que sintetizan lo tratado en clase. Es útil para ayudar a extraer las ideas más importantes de cada tema.

4) Actividades y evidencias. En esta parte se guardan todos los ejercicios y trabajos realizados, organizados por fecha y tema. Esto último permite comprobar el grado de estructuración y comprensión 
El portafolio como recurso didáctico en el proceso de aprendizaje del alumnado con trastorno por déficit de atención/hiperactividad

Ma Teresa Martínez-Frutos y Eva Herrera-Gutiérrez

de los contenidos y, por tanto, si se han alcanzado los objetivos de aprendizaje. Facilita la autocorrección y el seguimiento y evaluación por el docente.

5) Reflexiones y recomendaciones. Es importante que el estudiante realice una reflexión sobre lo que ha aprendido, las tareas que ha realizado para ello, las dificultades que ha tenido, para qué le sirve lo aprendido y todo aquello que considere oportuno resaltar. De este modo, al tiempo que se promueve la construcción personal del conocimiento, el alumno es más consciente de su proceso de aprendizaje y de cómo mejorarlo, lo que puede ser útil para planificar futuras actividades.

6) Progresos y logros. Es un apartado específico destinado a recoger los avances y logros del estudiante, lo que puede resultar muy positivo para conseguir mejorar su autoestima y comportamiento. No podemos olvidar que los problemas en estas áreas suelen estar en el origen de muchas de las dificultades académicas del menor con TDAH.

7) Calendario y planificación de tareas. Es el lugar idóneo donde apuntar los ejercicios para realizar en casa, los trabajos pendientes y las fechas de exámenes y otros eventos de interés. Se trata de indicar aquí las respuestas a las preguntas: qué tengo que hacer y cómo. Esta sección ayuda a recordar y supervisar las tareas pendientes y a organizarse mejor.

8) Conclusiones, autoevaluación y valoración general. Apartado final destinado a reflejar las conclusiones más importantes, la autoevaluación general y la valoración global de todo el proceso.

En cualquier caso, conviene tener presente que las diferentes secciones del portafolio deben acomodarse a las necesidades concretas del alumnado, a su edad y a las características de las materias para las que se destine.

Esto es algo que contemplan las modernas propuestas de portafolio digital o electrónico (Barberá \& de Martín, 2009; Rey \& Escalera, 2011), donde todas las fases de diseño y elaboración del mismo tienen que ir acompañadas del seguimiento y apoyo del docente, quien se ha de encargar de orientar al estudiante para que realice las oportunas correcciones y ajustes.

El portafolio digital, frente al tradicional o clásico, cuenta con la ver- 
satilidad del medio digital, la facilidad de almacenar información, ampliar o modificar los documentos de trabajo y compartirlos con otros sin limitaciones espacio-temporales. Además es un recurso que promueve especialmente el desarrollo de ciertas competencias (capacidad para comunicarse en medios variados; para interpretar, evaluar y usar la información de fuentes diversas...) y el aprendizaje activo e interactivo, posibilitando al alumno un feedback inmediato sobre la consecución de los objetivos y la eficacia de las estrategias que ha empleado en el proceso de aprendizaje.

\section{Conclusiones}

Falta de concentración, dificultades para la regulación del comportamiento, desajustes emocionales, problemas en las relaciones sociales y bajo rendimiento académico son algunas de las características que a menudo presentan los niños con trastorno hiperactivo, junto a una serie de cualidades y rasgos positivos de su personalidad que conviene aprovechar en los aprendizajes.

Realizar un rápido y acertado diagnóstico y una adecuada intervención desde diferentes perspectivas (médica, psicológica, educativa) es esencial, tanto para intentar paliar las consecuencias negativas del TDAH en el desarrollo del menor y en su entorno como para evitar la aparición posterior de otros trastornos (ansiedad, depresión, trastorno disocial, adicción a las drogas y un largo etcétera).

En el contexto del aula se deben fijar objetivos y emplear metodologías, estrategias y recursos didácticos que promuevan un desarrollo integral y favorezcan la resiliencia, resultando así beneficiosos para el conjunto de los alumnos y no solamente para el que presenta este trastorno.

De las utilidades principales del portafolio para el alumnado con TDAH destacamos su apoyo para organizar la información académica, estimular la creatividad y la motivación, promover hábitos de estudio, fomentar la responsabilidad, favorecer el aprendizaje consciente y autorregulado y mejorar el rendimiento escolar y la autoestima. Asimismo, puede ser útil al docente para diagnosticar las potencialidades y necesidades, los puntos fuertes y débiles, y de esta manera facilitar que los menores con esta alteración alcancen los objetivos curriculares. Al mismo tiempo, es una herramienta de trabajo que puede servir de fuente 
El portafolio como recurso didáctico en el proceso de aprendizaje del alumnado con trastorno por déficit de atención/hiperactividad

Ma Teresa Martínez-Frutos y Eva Herrera-Gutiérrez

de información y comunicación con los padres. Al permitirles conocer las tareas que deben realizar los niños y los contenidos que están aprendiendo a diario, pueden ayudarles a organizarse mejor y supervisar si realizan los trabajos.

En definitiva, el portafolio promueve una implicación más activa del alumno en el aprendizaje y le permite organizar y reflexionar sobre los contenidos académicos de forma más constante. Requiere una participación más planificada y creativa por parte del niño, por lo que contribuye a aumentar su motivación y mejorar su rendimiento académico. Además, posibilita que los maestros conozcan con mayor exactitud las posibilidades del alumno y la comunicación y colaboración con los padres.

\section{Referencias}

Aguilar-Domingo, M., Herrera-Gutiérrez, E., \& Gómez-Amor, J. (2013). Subtipos electrofisiológicos de personas diagnosticadas de TDAH. Características e implicaciones psicofisiológicas y educativas. Madrid: Editorial Académica Española. LAP LAMBERT Academic Publishing GmbH \& Co.

American Psychiatric Association (2000). Diagnostic and Statistical Manual of Mental Disorders, Fourth Edition, Text Revision (DSM-IV-TR). Washington, DC: Author (trad. cast. Barcelona: Masson, 2002).

American Psychiatric Association (2013). Diagnostic and Statistical Manual of Mental Disorders, Fifth Edition (DSM-5). Arlington, VA: Author (trad. cast. Madrid: Médica Panamericana, 2014).

Arcia, E., Frank, R., Sánchez-LaCay, A., \& Fernández, M. (2000). Teacher understanding of ADHD as reflected in attributions and classroom strategies. Journal of Attention Disorders, 4(2), 91-101.

Artiles, C. \& Jiménez, J.E. (2006). Escolares con trastorno por Déficit de Atención con o sin Hiperactividad (TDAH). Orientaciones para el profesorado. Canarias: Consejería de Educación, Cultura y Deportes, Gobierno de Canarias. Recuperado de http://sauce.pntic.mec.es/falcon/progtdah.pdf

Barberá, E. \& de Martín, E. (2009). Portfolio electrónico: aprender a evaluar el aprendizaje. Barcelona: UOC.

Barkley, R.A. (2002). Niños hiperactivos: cómo comprender y atender sus necesidades especiales ( $\left.2^{\mathrm{a}} \mathrm{ed}.\right)$. Barcelona: Paidós.

Barkley, R.A. (2005). ADHD and the nature of self-control (2nd ed.). Nueva York: Guilford Press.

Barkley, R.A. (2006). Attention deficit hyperactivity disorder: A handbook for diagnosis and treatment (3rd ed.). Nueva York: Guilford Press.

Barkley, R.A. (2008). El manejo del TDAH en el aula: estrategias para el éxito. Ponencia presentada en el II Congreso Nacional de TDAH, Madrid. 
El portafolio como recurso didáctico en el proceso de aprendizaje del alumnado con

trastorno por déficit de atención/hiperactividad Ma Teresa Martínez-Frutos y Eva Herrera-Gutiérrez

Barkley, R.A. (2010). Deficient emotional self-regulation is a core component of ADHD. Journal of ADHD and Related Disorder, 1(2), 5-37.

Cassany, D. (2002). El Portafolio Europeo de Lenguas. Aula de Innovación Educativa, 117, 13-17.

Cassany, D. (Ed.) (2006). El Portfolio Europeo de las Lenguas y sus aplicaciones en el aula. Madrid: Ministerio de Educación y Ciencia. Instituto Superior de Formación del Profesorado. Recuperado de http://books.google.es/books?id=qpazRE CzFToC\&pg=PA38\&lpg=PA38\&dq=el+portafolio+y+el+mcer \&source=bl\&ots= 8PyuU63OSm\&sig=s9zFyWkiLbVEYRIBFKOKad_y1Gk\&hl=es\&sa=X\&ei=ss1 Uq2EAeKJ7AaNo4HgCw\&ved=0CH0Q6AEwCQ\#v=onepage\&q=el\%20portafolio $\% 20 y \% 20$ el $\% 20$ mcer $\& \mathrm{f}=$ false

Colas, M.P. (2005). Portafolios y desarrollo de competencias profesionales en el marco del EEES. Revista de Ciencias de la Educación, 204, 1-20.

Consejería de Sanidad y Consumo - Consejería de Educación, Formación y Empleo, Región de Murcia (2012). Protocolo de Coordinación de Actuaciones Educativas y Sanitarias en la Detección y Diagnóstico del Trastorno por Déficit de Atención e Hiperactividad TDA-H (ed. rev.). Murcia: Consejería de Sanidad y Consumo. Servicio Murciano de Salud. Subdirección General de Salud Mental. Recuperado de http:// www.murciasalud.es/recursos/ficheros/236108-protocolo_2012.pdf

Eisenberg, D. \& Schneider, H. (2007). Perceptions of academic skills of children diagnosed with ADHD. Journal of Attention Disorders, 10(4), 390-397.

Gamo, J.R. (2012). La neuropsicología aplicada a las ciencias de la educación. Una propuesta que tiene como objetivo acercar al diálogo pedagogía/didáctica, el conocimiento de las neurociencias y la incorporación de las tecnologías como herramientas didácticas válidas en el proceso de enseñanza-aprendizaje. En J. Navarro, M.T. Fernández, F.J. Soto, \& F. Tortosa (Coords.), Respuestas flexibles en contextos educativos diversos. Murcia: Consejería de Educación, Formación y Empleo. Recuperado de http://diversidad.murciaeduca.es/publicaciones/dea2012/docs/jrgamo. pdf

García, E. (2000). Algunas aplicaciones del portafolio en el ámbito educativo. México: Secretaria de Educación y Cultura del Estado de Chihuahua.

González, A. (2015). Claves pedagógicas para la mejora de la calidad del EEES. Educatio Siglo XXI, 33(1), 259-276. doi: 10.6018/j/222591

González, C. (2014). Estrategias para trabajar la creatividad en la Educación Superior: pensamiento de diseño, aprendizaje basado en juegos y en proyectos. RED, Revista de Educación a Distancia, 40, 2-15. Recuperado de http://www.um.es/ead/red/40/ gonzalez.pdf

Herrera-Gutiérrez, E. (2015). Trastorno por Déficit de Atención con Hiperactividad: su investigación desde la perspectiva del desarrollo integral. Boletín de Investigación de la Facultad de Educación de la Universidad de Murcia, 2, 195-203. Recuperado de http://www.um.es/documents/299436/1725297/Boleti\%CC\%81n+de+Investigaci o\%CC\%81 n+Facultad+de+Educacio\%CC\%81 n+2015-1.pdf/b9e997e3-f3b0-4a24a02a-503546c $2 \mathrm{db} 17$

Herrera-Gutiérrez, E., Bermejo, R., Fernández, M.C., \& Soto, G. (2011). Déficit de aten- 
El portafolio como recurso didáctico en el proceso de aprendizaje del alumnado con trastorno por déficit de atención/hiperactividad

Ma Teresa Martínez-Frutos y Eva Herrera-Gutiérrez

ción e hiperactividad. En M.D. Prieto (Coord.), Psicología de la Excepcionalidad (pp. 139-152). Madrid: Síntesis.

Herrera-Gutiérrez, E., Calvo, M.T., \& Peyres, C. (2003). El trastorno por déficit de atención con hiperactividad desde una perspectiva actual. Orientaciones a padres y profesores. Revista de Psicología General y Aplicada, 56(1), 5-19.

Hidalgo, M.I. \& Soutullo, C. (2006). Trastorno por déficit de atención e hiperactividad (TDAH). Pediatría Integral, 6, 3-25.

Kahn, E. \& Cohen, L.H. (1934). Organic drivenners a brainstem syndrome and experience. New England Journal of Medicine, 5, 748-756.

Klenowski, V. (2005). Desarrollo de Portafolios para el aprendizaje y la evaluación. Procesos y principios ( $2^{\mathrm{a}}$ ed.). Madrid: Narcea.

Klimenko, O. (2008). La creatividad como un desafío para la educación del siglo XXI. Educación y Educadores, 11(2), 191-210.

Láez, M.C., Requejo, M., Silvano, J.J., \& Velasco, M.C. (2011). Protocolo de coordinación del trastorno por déficit de atención e hiperactividad. Valladolid: Gerencia Regional de Salud de la Junta de Castilla y León. Recuperado de http://www.saludcastillayleon. es/institucion/en/publicaciones-consejeria/buscador/protocolo-coordinacion-trastorno-deficit-atencion-hiperacti

Lasa, A. (2007). EI TDAH en el momento actual: controversias, divergencias y convergencias. Revista de Psicopatología y Salud Mental del Niño y del Adolescente, 2, 9-16.

Ley Orgánica 2/2006, de 3 de mayo, de Educación (LOE; BOE Núm. 106 de 4 de mayo de 2006).

Ley Orgánica 8/2013, de 9 de diciembre, para la Mejora de la Calidad Educativa (LOMCE; BOE Núm. 295 de 10 de diciembre de 2013).

Loe, I. \& Fieldman, H. (2007). Academic and educational outcomes of children with ADHD. Journal of Pediatric Psychology, 32(6), 643-654. Recuperado de http://jpepsy. oxfordjournals.org/content/32/6/643.full.pdf

López, B.S. \& Hinojósa, E.M. (2000). Evaluación del aprendizaje. Alternativas y nuevos desarrollos. México: Trillas.

Martínez-Frutos, M.T., Herrera-Gutiérrez, E., \& López-Ortuño, J. (2014). Conocimientos y lagunas de los docentes sobre el TDAH: la importancia de la formación. En J. Navarro, M.D. Gracia, R. Lineros, \& F.J. Soto (Coords.), Claves para una educación diversa. Murcia: Consejería de Educación, Cultura y Universidades. Recuperado de http:// diversidad.murciaeduca.es/publicaciones/claves/doc/mtmartinez.pdf

Miranda, A. (2011). Manual práctico de TDAH. Madrid: Síntesis.

Miranda, A., Presentación, M.J., Colomer, C., \& Roselló, B. (2011). Satisfacción con la vida de niños con trastorno por déficit de atención/hiperactividad: estudio de posibles factores de riesgo y de protección. Revista de Neurología, 52(1), 119-126.

Montoya, E. \& Herrera-Gutiérrez, E. (2014). Manifestaciones del TDAH en la etapa de educación infantil y cómo afrontarlas. En J. Navarro, M.D. Gracia, R. Lineros, \& F.J. Soto (Coords.), Claves para una educación diversa. Murcia: Consejería de Educación, Cultura y Universidades. Recuperado de http://diversidad.murciaeduca.es/publicaciones/claves/doc/emontoya.pdf

Mulas, F., Mattos, L., de la Osa-Langreo, A., \& Gandía-Benetó, R. (2007). Trastorno por 
El portafolio como recurso didáctico en el proceso de aprendizaje del alumnado con

trastorno por déficit de atención/hiperactividad Ma Teresa Martínez-Frutos y Eva Herrera-Gutiérrez

déficit de atención/hiperactividad: a favor del origen orgánico. Revista de Neurología, 44(Supl. 3), S47-S49.

Orden ECD/65/2015, de 21 de enero, por la que se describen las relaciones entre las competencias, los contenidos y los criterios de evaluación de la educación primaria, la educación secundaria obligatoria y el bachillerato (BOE Núm. 25 de 29 de enero de 2015).

Orjales, I. (2002). Déficit de atención con hiperactividad. Manual para padres y educadores ( $7^{\mathrm{a}} \mathrm{ed}$.). Madrid: CEPE.

Orjales, I. (2007). El tratamiento cognitivo en niños con trastorno por déficit de atención e hiperactividad (TDAH): revisión y nuevas aportaciones. Anuario de Psicología Clínica y de la Salud, 3, 19-30.

Rey, E. \& Escalera, A.M. (2011). El portafolio digital un nuevo instrumento de evaluación. Didáctica, Innovación y Multimedia, 21, 1-10. Recuperado de http://www.raco.cat/ index.php/DIM/article/view/247586/331525

Ross, D.M. \& Ross, S.A. (1982). Hyperactivity: Current issues, research and theory (2nd ed.). New York: Wiley.

Rutter, M. (1989). Isle of Wight revisited, twenty five years of child psychiatric epidemiology special article. Journal of the American Academy of Child and Adolescent Psychiatry, 28(5), 633-653.

Sánchez-Mármol, D.J. \& Herrera-Gutiérrez, E. (2010). El alumno hiperactivo y su funcionamiento en el ámbito académico. En A. de Pro Bueno (Coord.), Investigación e Innovación en Educación Infantil y Educación Primaria, II (pp. 579-598). Murcia: Facultad de Educación. Universidad de Murcia. Recuperado de http://www.um.es/c/document library/get_file?uuid=1867c6b6-3023-4590-9831-3fe19ed2d347 \&groupld=299436

Scandar, R. (2000). El niño que no podía dejar de portarse mal. Buenos Aires: Distal.

Sharma, A. \& Couture, J. (2014). A review of the pathophysiology, etiology, and treatment of attention-deficit hyperactivity disorder (ADHD). Annals of Pharmacotherapy, 48(2), 209-225. doi: 10.1177/1060028013510699

Soutullo, C. (2004). Convivir con niños y adolescentes con trastorno por déficit de atención e hiperactividad. Madrid: Médica Panamericana.

Strauss, A. \& Lehtinen, L.E. (1947). Psychopathology and education of the brain-injured child. New York: Grune and Stratton.

Tirapu, J., Muñoz, J.M., Pelegrín, C., \& Albéniz, A. (2005). Propuesta de un protocolo para la evaluación de las funciones ejecutivas. Revista de Neurología, 41(3), 177-186. Wender, P. (1971). Minimal brain dysfunction in children. New York: Wiley and Sons. 
